\title{
THE CRAB PULSAR EMISSION: GIANT PULSES AND A POSSIBLE PRECURSOR TO THE INTERPULSE
}

\author{
JEFFREY FOSTER FRIEDMAN \\ University of Puerto Rico at Mayagüez, and Oklahoma University \\ VALENTÍN BorIAKOFF \\ Worcester Polytechnic Institute
}

\begin{abstract}
We have examined approximately 550,000 pulses from PSR $0531+21$ and have detected 1814 giant pulses $6 \sigma$ or more above the background noise level. We hae measured the relative arrival times and the energies of both the main pulse and interpulse, and have found that the giant pulses arrive somewhat earlier than the peaks in the average profile. Approximately $7 \%$ of the main pulse and $1 \%$ of the interpulse energy is in the form of giant pulses $8 \sigma$ more above the background level. No giant pulses have been detected in the precursor at the $8 \sigma$ level. In addition we have found a slight, although apparently real, rise in emission in the region where an interpulse precursor would be if the emission is symmetric.
\end{abstract}

\section{Introduction}

Giant radio pulses emitted by the Crab pulsar led to its discovery by Staelin and Reifenstein (1968). Since then several studies at different frequencies have refined our knowledge of these giant pulses and their energy characteristics (Staelin and Sutton 1970, Heiles, Campbell, and Rankin 1970, Heiles and Rankin 1971, Argyle and Gower 1972, Gower and Argyle 1972, Linscott and Hankins 1981). General rate and energy statistics are known at several frequencies, but the resolution and numbers are usually low. We have both good time resolution ( $\approx 20 \mu$ s using fitting routines), and signal-tonoise ratio $>6$ using an on-line dedisperser (Boriakoff 1974), and the Arecibo 305-meter telescope at $430 \mathrm{MHz}$. We have a large sample of pulses with accurate arrival times and good peak energy resolution. Because of a small, but significant spurious detection rate, and an apparent discrepancy in the relative number of giant pulses at the $6 \sigma$ and $8 \sigma$ levels, we have chosen to concentrate on those giant pulses $>8 \sigma$ above the background. We have also constructed average profiles for the giant pulses and for the general pulsed emission.

\section{Observational parameters}

PSR $0531+21$ was observed at $430 \mathrm{MHz}$ using a circularly polarized feed. A $32 \times 20 \mathrm{kHz}$ filter bank followed by an on-line post-detection dedisperser was used. The data were smoothed by $10 \mu$ s, then synchronously sampled at $80 \mu$ s intervals in two 5-ms windows centered on the main pulse and interpulse, for a total of 126 samples per pulse period.

\section{Arrival times}

In figure 1 we present the average profiles of all of the data and the average profile of the subset of data containing only the giant pulses. The giantpulse average profile peaks about $65 \mu$ s earlier for the main pulse and $70 \mu \mathrm{s}$ for the interpulse. A further breakdown shows that giant pulses with peak $\mathrm{S} / \mathrm{N}>12 \sigma$ arrive earlier than weaker pulses, a result is confirmed by graphical, arithmetic and Gaussian curve fitting analysis.

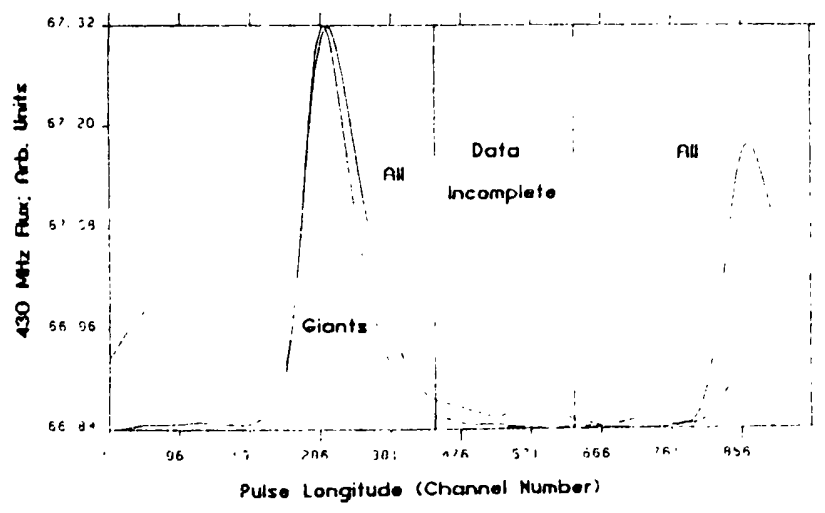

Figure 1 Upper trace: Average of all pulses. Lower trace: Average profile of giant pulses. The abscissa scale is 11.5 channel number units per degree of pulse phase.

\section{Energy}

Gower and Argyle (1972) reported that $0.7 \%$ of the pulsed energy in their $146 \mathrm{MHz}$ sample is attributable to giant pulses. Using their suggested scaling law (Argyle and Gower 1972), $n \propto S^{-2.5}$, where $n$ is the number of pulses at flux $S$, they 
would have only a few percent of the energy coming from pulses $\geq 10$ times the average pulse amplitude. We found that $7 \%(1 \%)$ of the main pulse (interpulse) energy comes from our $>8 \sigma$ sample. Using the same scaling law we find $26 \%$ of the main pulse energy is from pulses greater than 10 times the average pulse. Linscott and Hankins (1985) have found an even more extreme case at $1400 \mathrm{MHz}$, where they report a relative incidence of $10^{-3}$ for giant pulses with energies $10^{5}$ average, indicating almost all of the pulsed energy coming from the giant pulses. This implies emission from essentially two different modes, one almost a null state. It may also imply a different scaling law, or different production mechanisms at different energies, and/or frequencies. We have made a power-law fit to our data, and we find the exponent $(-2.5)$ used by Gower and Argyle to be reasonable overall, but seriously discrepant over more restricted ranges in the data. We find three separate power law regimes, with exponents $-3,-2,-1$, from low to high energy pulses. Table 1 summarizes our energy data and gives the number of pulses found in the main pulse and interpulse as a function of height of the pulses above the background.

Table 1

\begin{tabular}{|lrrrrrrrrrr|}
\hline \multicolumn{1}{|c|}{$6 \sigma$} & $8 \sigma$ & $10 \sigma$ & $12 \sigma$ & $14 \sigma$ & $16 \sigma$ & $18 \sigma$ & $20 \sigma$ & $22 \sigma$ & $24 \sigma$ \\
\hline$N_{\text {tot }}$ & 1814 & 763 & 441 & 304 & 217 & 167 & 135 & 114 & 104 & 93 \\
$N_{\text {mp }}$ & 1579 & 665 & 386 & 275 & 195 & 153 & 122 & 104 & 95 & 85 \\
$N_{\text {ip }}$ & 235 & 98 & 55 & 29 & 22 & 14 & 13 & 10 & 9 & 8 \\
$N_{\text {ip }} / N_{\text {mp }}$ & 149 & .147 & .143 & .106 & .113 & .092 & .107 & .096 & .095 & .094 \\
$\mathrm{MP} \%$ & 87 & 87 & 88 & 91 & 90 & 92 & 90 & 91 & 91 & 91 \\
\hline
\end{tabular}

\section{The interpulse precursor?}

In the course of this investigation we constructed an average pulse profile using most of our data by matching the peaks of our individual session average profiles, approximately 25000 pulses per profile, and summing. Peak positions were determined using a fitting routine and partial bin offsets were compensated for by linear interpolation. The result, shown in figure 2, was surprising, so we tried the same approach with several subsets of the data, with similar results. We believe the rise in emission in the region where one would expect the interpulse precursor to be real, and have tentatively identified it as the precursor to the interpulse.

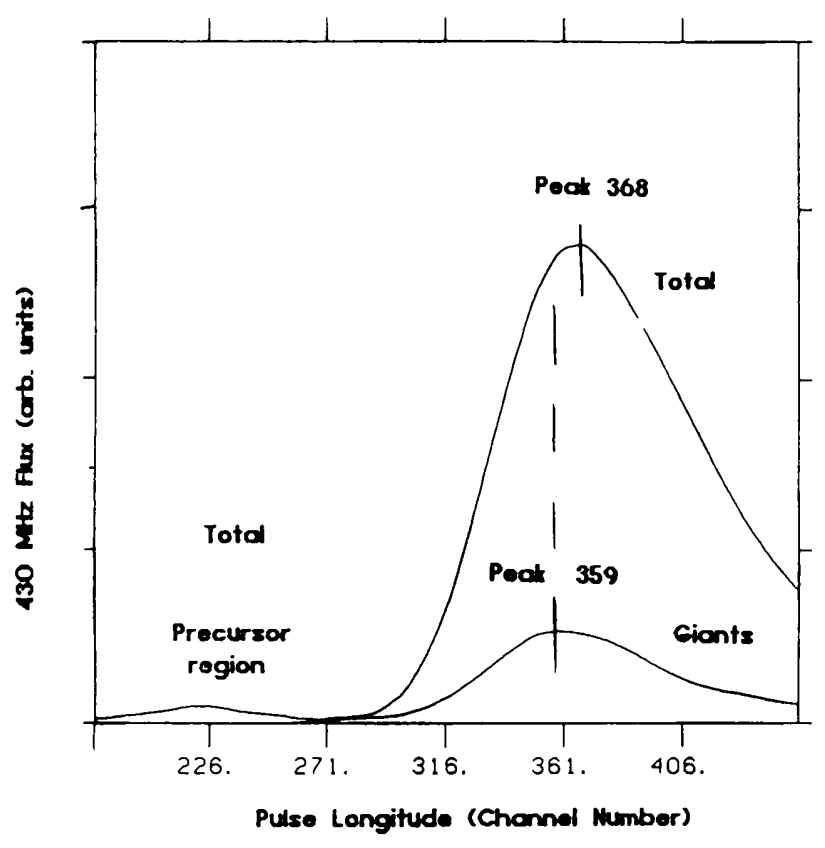

Figure 2 Upper trace: Average profile of 555000 periods. Lower trace: Average profile of the giant interpulses. The plot shows the possible interpulse precursor and the earlier arrival time of the giant interpulses, relative to the grand average profile peak. The abscissa scale is 11.5 channel number units per degree of pulse phase.

\section{Conclusions}

The emission of pulsed energy from the Crab pulsar appears to be different in the normal and giant modes. The giant pulses in our sample occur earlier than the average profile peak, and the scaling law with frequency may be different for the giant and normal pulses. More of the pulsed energy seems to come from the larger pulses at higher frequencies. We have also discovered what we tentatively identify as a precursor to the interpulse. There seems to be a real rise in emission when we add our entire sample in phase by alignment of profile peaks.

Acknowledgment: This work has been supported in part by the Arecibo Observatory, which is part of the National Astronomy and Ionosphere Center, operated by Cornell University under a management agreement with the National Science Foundation. 